Pacific Journal of Mathematic 


\title{
SHADOW AND INVERSE-SHADOW INNER PRODUCTS FOR A CLASS OF LINEAR TRANSFORMATIONS
}

\author{
GeORge Golightly
}

\begin{abstract}
Suppose $\{H,(\cdot, \cdot)\}$ is a complete inner product space and $H_{1}$ is a dense subspace of $H$. In case $T$ is a linear transformation from $H_{1}$ to $H_{1}$ (perhaps not bounded), a necessary and sufficient condition is obtained in Theorem 1 for the existence of an inner product $(\cdot, \cdot)_{1}$ for $H_{1}$ such that (i) the identity is continuous from $\left\{H_{1},(\cdot, \cdot)_{1}\right\}$ to $\{H,(\cdot, \cdot)\}$ and (ii) $T$ is bounded in $\left\{H_{1},(\cdot, \cdot)_{1}\right\}$. When this condition holds, the inverse-shadow inner product is defined on $H_{1}$, for sufficiently large positive numbers $\beta$, by $(x, y)_{\beta, T}=\sum_{p=0}^{\infty}\left((T / \beta)^{p} x,(T / \beta)^{p} y\right)$. An extension of Theorem 1 provides a necessary and sufficient condition for the existence of an inner product $(\cdot, \cdot)_{1}$ for $H_{1}$ such that $\left\{H_{1},(\cdot, \cdot)_{1}\right\}$ is complete and (i) and (ii) hold. This latter condition, stated in Theorem 5 in terms of a pair of inverse-shadow inner products, depends on a description of those complete inner product spaces $\left\{H_{1},(\cdot, \cdot)_{1}\right\}$, with $H_{1}$ dense in $H$, for which (i) holds. According to this description, given in Theorem 4 , each such inner product $(\cdot, \cdot)_{1}$ is a scalarmultiple of an inverse-shadow inner product $(\cdot, \cdot)_{\delta, c}$, where $C$ is a bounded operator on $H$ mapping $H_{1}$ to $H_{1}$ and $\delta=1$.
\end{abstract}

This pattern was developed in an investigation, other results of which are in [4]. If $H_{1}$ is a linear subspace of $H,(\cdot, \cdot)_{1}$ is an inner product for $H_{1}$, and the identity is continuous from $\left\{H_{1},(\cdot, \cdot)_{1}\right\}$ to $\{H,(\cdot, \cdot)\},\left\{H_{1},(\cdot, \cdot)_{1}\right\}$ is said in [6] to be continuously situated in $\{H,(\cdot, \cdot)\}$. The setting in Theorem 4 of a pair of complete inner product spaces, one continuously situated in the other, is discussed in [1], [2], [6], and [7]. Additional results in Theorems 2 and 3 relate the shadow inner product, the inner product $\left(\left(1-T^{*} T / \beta^{2}\right) \cdot, \cdot\right)^{\prime}$ in those theorems, and the inverse-shadow inner product $(\cdot, \cdot)_{\beta, T}$. In contrast to Theorem 4, an example at the end of the paper shows that $\left\{H_{1},(\cdot, \cdot)_{\beta, T}\right\}$ may be complete even when the closure in $H \times H$ of $T$ is not a function.

Here is an example to which Theorem 1 applies (with $H=H_{1}$ ). Start with a complete infinite dimensional inner product space $\left\{H^{\prime},(\cdot, \cdot)^{\prime}\right\}$, a one-to-one (continuous) operator $T$ on $H^{\prime}$ with range a dense, proper subspace of $H^{\prime}$, and a closed subspace $Z$ of $H^{\prime}$ such that $Z \cap T\left(H^{\prime}\right)$ is $\{0\}$. Now, with $P$ the orthogonal projection of $H^{\prime}$ onto $Z^{\perp}$, there is, by the Axiom of Choice, an algebraic complement $H_{1}$ of $Z$ in $H^{\prime}$ of which $T\left(H^{\prime}\right)$ is a subspace and, with $(\cdot, \cdot)$ the inner product on $H_{1}$ such that $(x, y)=(P x, P y)^{\prime},\left\{H_{1},(\cdot, \cdot)\right\}$ is com- 
plete and for $x$ in $H_{1}(x, x) \leqq(x, x)^{\prime}$. Yet the restriction of $T$ to $H_{1}$ is not continuous in $\left\{H_{1}(\cdot, \cdot)\right\}$. Of course, the above construction uses the Axiom of Choice, as the result of [8] implies it must. However, this use is not in constructing $T$ but in selecting the subspace $H_{1}$ of $H^{\prime}$.

Throughout the paper, $\{H,(\cdot, \cdot)\}$ is a complete infinite dimensional inner product space and $H_{1}$ a dense subspace of $H$. If some variation of the symbols ' $(\cdot, \cdot)$ ' denotes an inner product for the space $S$, then the corresponding variation of ' $\|\cdot\|$ ' denotes the corresponding norm for $S$. For instance, $\|x\|_{\beta, T}=\left[(x, x)_{\beta, T}\right]^{1 / 2}$. An operator on $\{H,(\cdot, \cdot)\}$ is a continuous linear transformation from all of $H$ to (into) $H$. A closed operator in $\{H,(\cdot, \cdot)\}$ is a linear transformation from a dense subspace of $H$ to $H$ whose graph is closed in $H \times H$. If $Z$ and $Z^{\prime}$ are two subspaces of $H$ such that $Z \cap Z^{\prime}$ is $\{0\}$ and $H$ is the linear span of $Z$ and $Z^{\prime}$, then $Z$ is said to be an algebraic complement in $H$ of $Z^{\prime}$ and that linear transformation $\phi$ on $H$ such that $\phi$ is the identity 1 on $Z$ and 0 on $Z^{\prime}$ is called the algebraic projection of $H$ onto $Z$ with kernel $Z^{\prime}$. If $Z$ is a subset of $H, \bar{Z}$ is the closure of $Z$ in $H$.

\section{THEOREMS AND EXAMPLES}

THeORem 1. Suppose that $T$ is a linear transformation from $H_{1}$ to $H_{1}$. In order that there be a norm $\|\cdot\|_{1}$ for $H_{1}$ such that (i) there is a positive number $c$ such that $\|\cdot\| \leqq c\|\cdot\|_{1}$ on $H_{1}$ and (ii) $T$ is continuous in $\left\{H_{1},\|\cdot\|_{1}\right\}$ it is necessary and sufficient that there be a positive number $\beta$ such that for $x$ in $H_{1} \sum_{p=0}^{\infty}\left\|(T / \beta)^{p} x\right\|^{2}$ converges. In case there is such a norm $\|\cdot\|_{1}$, if $\beta$ is a number exceeding the operator-norm for $T$ in $\left\{H_{1},\|\cdot\|_{1}\right\}$ then for $x$ and $y$ in $H_{1}$ the formula $(x, y)_{\beta, T}=\sum_{p=0}^{\infty}\left((T / \beta)^{p} x,(T / \beta)^{p} y\right)$ defines an inner product $(x, y)_{\beta, T}$ for $H_{1}$ such that

(1) there is a positive number $d$ such that for $x$ in $H_{1}\|x\| \leqq$ $\|x\|_{\beta, T} \leqq d\|x\|_{1}$

(2) for $x$ in $H_{1} \lim _{p \rightarrow \infty}\left\|(T / \beta)^{p} x\right\|_{\beta, T}=0$, and

(3) for $x$ and $y$ in $H_{1}(T x, T y)_{\beta, T}=\beta^{2}\left[(x, y)_{\beta, T}-(x, y)\right]$.

Proof. In case there is a positive number $\beta$ for which $\sum_{p=0}^{\infty}\left\|(T / \beta)^{p} x\right\|^{2}$ converges on $H_{1}$, we have for $x$ and $y$ in $H_{1}$ and $n$ a positive integer,

$$
\begin{aligned}
& \sum_{p=0}^{n}\left|\left((T / \beta)^{p} x,(T / \beta)^{p} y\right)\right| \\
& \quad \leqq \sum_{p=0}^{n}\left\|(T / \beta)^{p} x\right\|\left\|(T / \beta)^{p} y\right\|
\end{aligned}
$$




$$
\leqq\left(\sum_{p=0}^{n}\left\|(T / \beta)^{p} x\right\|^{2}\right)^{1 / 2}\left(\sum_{p=0}^{n}\left\|(T / \beta)^{p} y\right\|^{2}\right)^{1 / 2},
$$

so that $\sum_{p=0}^{\infty}\left((T / \beta)^{p} x,(T / \beta)^{p} y\right)$ converges absolutely. Moreover, the formula $(x, y)_{\beta, T}=\sum_{p=0}^{\infty}\left((T / \beta)^{p} x,(T / \beta)^{p} y\right)$ defines as inner product for $H_{1}$.

Suppose that there is a norm $\|\cdot\|_{1}$ for $H_{1}$ for which (i) and (ii) hold. Suppose $n$ is a positive integer, $\beta$ is a positive number, and $r$ is a number greater than 1 such that for $x$ in $H_{1} r\|T x\|_{1} \leqq \beta\|x\|_{1}$. Then for $x$ and $y$ in $H_{1}$

$$
\begin{aligned}
& \sum_{p=0}^{n}\left|\left((T / \beta)^{p} x,(T / \beta)^{p} y\right)\right| \\
& \quad \leqq \sum_{p=0}^{n}\left\|(T / \beta)^{p} x\right\|\left\|(T / \beta)^{p} y\right\| \\
& \leqq c^{2} \sum_{p=0}^{n}\left\|(T / \beta)^{p} x\right\|_{1}\left\|(T / \beta)^{p} y\right\|_{1} \\
& \leqq c^{2} \sum_{p=0}^{n}\|x\|_{1}\|y\|_{1}\left(1 / r^{2 p}\right) \\
& \quad=c^{2}\|x\|_{1}\|y\|_{1} r^{2} /\left(r^{2}-1\right) .
\end{aligned}
$$

Thus, for $x$ and $y$ in $H_{1}$ the series $\sum_{p=0}^{\infty}\left((T / \beta)^{p} x,(T / \beta)^{p} y\right)$ converges absolutely and, replacing $y$ by $x$ in (A), we have

$$
\sum_{p=0}^{n}\left\|(T / \beta)^{p} x\right\|^{2} \leqq c^{2}\left(\|x\|_{1}\right)^{2} r^{2} /\left(r^{2}-1\right) .
$$

Note that (1) follows from (B) with $d=c r /\left(r^{2}-1\right)^{1 / 2}$. To establish (2), observe that for $x$ in $H_{1}$

$$
\begin{gathered}
\left(\left\|(T / \beta)^{p} x\right\|_{\beta, T}\right)^{2}=\sum_{q=0}^{\infty}\left\|(T / \beta)^{p+q} x\right\|^{2} \longrightarrow 0 \\
\text { as } p \longrightarrow \infty,
\end{gathered}
$$

since $\sum_{q=0}^{\infty}\left\|(T / \beta)^{q} x\right\|^{2}$ converges. The equality (3) is established by noting that

$$
\begin{aligned}
(T x, & T y)_{\beta, T} \\
& =\sum_{p=0}^{\infty}\left((T / \beta)^{p} T x,(T / \beta)^{p} T y\right) \\
& =\beta^{2} \sum_{p=1}^{\infty}\left((T / \beta)^{p} x,(T / \beta)^{p} y\right) \\
& =\beta^{2}\left[\sum_{p=0}^{\infty}\left((T / \beta)^{p} x,(T / \beta)^{p} y\right)-(x, y)\right] \\
& =\beta^{2}\left[(x, y)_{\beta, T}-(x, y)\right] .
\end{aligned}
$$

The following example is offered in connection with Lemma 1. This lemma is useful in the proof of Theorems 3 and 4 . 
EXAMPLE 1. Suppose that $S$ is the subspace of $L^{2}[0,1]$ of all absolutely continuous $f$ on $[0,1]$ such that $f^{\prime}$ is in $L^{2}[0,1]$ and for such $f T f=f^{\prime}$, so that $T$ is a closed operator in $L^{2}[0,1]$. Suppose $H_{1}$ is the set of all $f$ in $S$ such that for $p \geqq 0 \quad T^{p} f$ is in $S$ and $\sum_{p=0}^{\infty} \int_{0}^{1}\left|T^{p} f\right|^{2}$ converges. Then $H_{1}$ is a dense subspace of $L^{2}[0,1]$ and, with $\beta=1$ and $(f, g)_{\beta, T}=\sum_{p=0}^{\infty} \int_{0}^{1}\left[T^{p} f\right]\left[T^{p} g\right]^{*}$ on $H_{1},\left\{H_{1},(\cdot, \cdot)_{\beta, T}\right\}$ is complete.

Lemma 1. Suppose that $T$ is a closed operator in $\{H,(\cdot, \cdot)\}$ and $\beta>0$. Then the set $H_{2}$ of all $x$ in $H$ such that for $p>0 x$ is in the domain of $T^{p}$ and $\sum_{p=0}^{\infty}\left\|(T / \beta)^{p} x\right\|^{2}$ converges is a linear space such that $T\left(H_{2}\right)$ lies in $H_{2}$. Also, if $(\cdot, \cdot)_{\beta, T}$ is the inner product for $H_{2}$ given, as in Theorem 1 , by $(x, y)_{\beta, T}=\sum_{p=0}^{\infty}\left((T / \beta)^{p} x,(T / \beta)^{p} y\right)$ then $\left\{H_{2},(\cdot, \cdot)_{\beta, T}\right\}$ is complete. In case $T$ is self-adjoint in $\{H,(\cdot, \cdot)\}$, then the restriction of $T$ to $H_{2}$ is self-adjoint in $\left\{H_{2},(\cdot, \cdot)_{\beta, T}\right\}$.

The following argument is offered. In general (when $T$ is only closed and not defined everywhere), $H_{2}$ need not be dense in $H$. Suppose $x$ is in $H_{2}$. Then $\sum_{p=0}^{\infty}\left\|(T / \beta)^{p} T x\right\|^{2}=\beta^{2} \sum_{p=1}^{\infty}\left\|(T / \beta)^{p} x\right\|^{2}$, so that $T x$ is in $H_{2}$. To show that $H_{2}$ is a linear space, suppose $S_{1}$ is the linear space of all $H$-valued sequences, $S_{2}$ is the subspace of $S_{1}$ to which $z$ belongs only in case $\sum_{p=0}^{\infty}\left\|z_{p}\right\|^{2}$ converges, and for $z$ and $w$ in $S_{2}\langle z, w\rangle=\sum_{p=0}^{\infty}\left(z_{p}, w_{p}\right)$, so that $\left\{S_{2},\langle\cdot, \cdot\rangle\right\}$ is a complete inner product space. Suppose $D$ is the set of all $x$ in $H$ such that for $p>0 x$ is in the domain of $T^{p}$ and $\widetilde{T}$ the linear transformation from $D$ to $S_{1}$ such that for $p \geqq 0(\widetilde{T} x)_{p}=(T / \beta)^{p} x$. Note that $H_{2}=\widetilde{T}^{-1}\left(S_{2}\right)$, a linear space, and that $\widetilde{T}$, restricted to $H_{2}$, is a linear isometry from $\left\{H_{2},(\cdot, \cdot)_{\beta, T}\right\}$ onto a subspace of $S_{2}$. Suppose $y$ is a convergent sequence in $\left\{H_{2},(\cdot, \cdot)_{\beta, T}\right\}$. Then $\widetilde{T} y$ is convergent in $S_{2}$, with limit $z$ in $S_{2}$. Since, for $p \geqq 0$ the sequence $\left\{(T / \beta)^{p} y,(T / \beta)^{p+1} y\right\}$ has values in the closed transformation $T / \beta$ and limit $\left\{z_{p}, z_{p+1}\right\}$ in $H \times H, z_{p+1}=$ $(T / \beta) z_{p}$. Thus, for $p \geqq 0 z_{p}=(T / \beta)^{p} z_{0}$, so that $z=\widetilde{T} z_{0}$. Since $\widetilde{T}$ is an isometry, $y$ has limit $z_{0}$ in $\left\{H_{2},(\cdot, \cdot)_{\beta, T}\right\}$. Suppose $T$ is self-adjoint in $\{H,(\cdot, \cdot)\}$. Then for $x$ and $y$ in $H_{2}$

$$
\begin{aligned}
(T x, y)_{\beta, T} & =\sum_{p=0}^{\infty}\left((T / \beta)^{p} T x,(T / \beta)^{p} y\right) \\
& =\sum_{p=0}^{\infty}\left((T / \beta)^{p} x,(T / \beta)^{p} T y\right)=(x, T y)_{\beta, T},
\end{aligned}
$$

so that $T$ is self-adjoint on the complete space $\left\{H_{2},(\cdot, \cdot)_{\beta, T}\right\}$.

EXAMPLE 2. This example shows that in case $\{H,(\cdot, \cdot)\}$ is separable the set of linear transformations $T$ with domain $H$ and 
range lying in $H$ for which there is a positive number $\beta$ such that $\sum_{p=0}^{\infty}\left\|(T / \beta)^{p} x\right\|^{2}$ converges on $H$ is not a linear space.

Suppose $y$ is in $H,\|y\|=1$, and $Y$ is the linear span of $\{y\}$. Suppose $\left\{e_{m}\right\}_{1}^{\infty}$ is a complete orthonormal sequence in $H \ominus Y$. Suppose for $m>0 u_{m}=e_{m}+(m !) y$. The linear span $U$ of $\left\{u_{m}\right\}_{1}^{\infty}$ is dense in $H$. One sees this by noting that $y=\lim _{m \rightarrow \infty}\left(u_{m} / m !\right)$. Hence, for $p>0 e_{p}=u_{p}-(p !) y$ is in $\bar{U}$. Thus, the linear space $\bar{U}$ includes both $Y$ and $H \ominus Y$. Suppose that $Z$ is an algebraic complement of $Y$ in $H$ of which $U$ is a subspace. Suppose $\phi$ is the algebraic projection of $H$ onto $Z$ with kernel $Y$ and that $C$ is the operator on $H$ such that $C y=0$ and for $m$ a positive integer $C e_{m}=e_{m+1}$. Since the operator-norm of $C$ is $1, \sum_{p=0}^{\infty}\left\|(C / 2)^{p} x\right\|^{2}$ converges on $H$. Since for $p>0(\phi-1)^{p}=(-1)^{p+1}(\phi-1), \quad \sum_{p=0}^{\infty}\left\|[(\phi-1) / 2]^{p} x\right\|^{2}$ converges on $H$.

Suppose $T$ is $C+(\phi-1)$ and $m$ is the number-sequence such that $m_{1}=1$ and for $n>0 m_{n+1}=(n+1) !-m_{n}$. Then for $n>0$ $T^{n}\left(e_{1}\right)=e_{n+1}+m_{n} y$ and $\left\|T^{n} e_{1}\right\|^{2}=1+m_{n}^{2}$. Note that for $n \geqq 1$ $n !-(n-1) ! \leqq m_{n} \leqq n !$, so that $m_{n+1} \geqq n !$. Thus, for $\beta>0$ $\sum_{p=0}^{\infty}\left\|(T / \beta)^{p} e_{1}\right\|^{2}$ diverges.

THEOREM 2. Suppose that $\left\{H^{\prime},(\cdot, \cdot)^{\prime}\right\}$ is a complete inner product space, $T$ is an operator on $\left\{H^{\prime},(\cdot, \cdot)^{\prime}\right\}$, and $H_{1}$ is a dense subspace of $H^{\prime}$ such that $T\left(H_{1}\right)$ lies in $H_{1}$. Suppose, moreover, that there is $a$ positive number $\beta$ such that for each of $x$ and $y$ in $H_{1}(x, y)^{\prime}=$ $\sum_{p=0}^{\infty}\left((T / \beta)^{p} x,(T / \beta)^{p} y\right)$. Then (i) $\beta$ is not less than the operator-norm for $T$ in $\left\{H^{\prime},(\cdot, \cdot)^{\prime}\right\}$, (ii) with $T^{*}$ the adjoint of $T$ in $\left\{H^{\prime},(\cdot, \cdot)^{\prime}\right\}$ and $x$ and $y$ in $H_{1}(x, y)=\left(\left(1-T^{*} T / \beta^{2}\right) x, y\right)^{\prime}$, and (iii) in case $H^{\prime} \neq H_{1}$ and $\left\{H_{1},(\cdot, \cdot)\right\}$ is complete, so that $H=H_{1}$, then $\beta$ is the operator-norm for $T$ in $\left\{H^{\prime},(\cdot, \cdot)^{\prime}\right\}$ and for $T$ on $H_{1}$ in $\left\{H_{1},(\cdot, \cdot)^{\prime}\right\}$.

Proof. Since $H_{1}$ is dense in $H^{\prime}$ and $T$ continuous on $H^{\prime}$, the operator-norm for $T$ in $\left\{H^{\prime},(\cdot, \cdot)^{\prime}\right\}$ is the operator-norm for $T$ on $H_{1}$ in $\left\{H_{1},(\cdot, \cdot)^{\prime}\right\}$. Suppose that for $x$ and $y$ in $H_{1}(x, y)^{\prime}=\sum_{p=0}^{\infty}\left((T / \beta)^{p} x\right.$, $\left.(T / \beta)^{p} y\right)$. Then for $x$ in $H_{1}$

$$
\left(\|T x\|^{\prime}\right)^{2}=\beta^{2}\left[\left(\|x\|^{\prime}\right)^{2}-\|x\|^{2}\right] \leqq \beta^{2}\left(\|x\|^{\prime}\right)^{2} .
$$

Thus, $\beta$ is not less than the operator-norm for $T$ in $\left\{H^{\prime},(\cdot, \cdot)^{\prime}\right\}$. Also, on $H_{1}$

$$
\begin{aligned}
(x, y) & =(x, y)^{\prime}-((T / \beta) x,(T / \beta) y)^{\prime} \\
& =\left(\left(1-T^{*} T / \beta^{2}\right) x, y\right)^{\prime},
\end{aligned}
$$

so that (ii) is established.

To prove (iii), note that, since $H^{\prime} \neq H_{1}, H_{1}$ is not closed in $H^{\prime}$. 
Also, the identity function from $\left\{H_{1},(\cdot, \cdot)^{\prime}\right\}$ to $\left\{H_{1},(\cdot, \cdot)\right\}$ is continuous. Since $\left\{H_{1},(\cdot, \cdot)\right\}$ is complete, the identity function from $\left\{H_{1},(\cdot, \cdot)\right\}$ to $\left\{H_{1},(\cdot, \cdot)^{\prime}\right\}$ is not continuous. By the Closed Graph theorem, the set $Z$ of all $\|\cdot\|^{\prime}$-limits in $H^{\prime}$ of $H_{1}$-sequences having $\|\cdot\|$-limit 0 is nondegenerate. Since $Z$ is the kernel of $\left(1-T^{*} T / \beta^{2}\right)^{1 / 2}$, there is a nonzero point $x$ of $H^{\prime}$ such that $x=\left(T^{*} T / \beta^{2}\right) x$. Thus, $\left(\|T x\|^{\prime}\right)^{2}=\beta^{2}\left(\|x\|^{\prime}\right)^{2}$. In view of (i), (iii) is established.

REMARK. Here I will describe why I call an inner product, $\left(\left(1-T^{*} T / \beta^{2}\right) \cdot, \cdot\right)^{\prime}$, a shadow inner product. The point of view taken by the author is that one starts with $\{H,(\cdot, \cdot)\}$, a linear transformation $T$ from $H$ to $H$, not continuous in $\{H,(\cdot, \cdot)\}$, and a positive number $\beta$ such that $\sum_{p=0}^{\infty}\left\|(T / \beta)^{p} x\right\|^{2}$ converges on $H$. ( $T$ might be the transformation $\phi-1$ of Example 2 with $\beta=2$ ). One builds the space $\left\{H,(\cdot, \cdot)_{\beta, T}\right\}$ with a completion $\left\{H^{\prime}(\cdot, \cdot)^{\prime}\right\}$ so that $H$ is a proper subspace of $H^{\prime}$, dense in $H^{\prime}$. Now $T$ has continuous linear extension to $H^{\prime}$, also denoted by $T$, with adjoint $T^{*}$ in $\left\{H^{\prime},(\cdot, \cdot)^{\prime}\right\}$. Then by Theorem $2,(x, y)=\left(\left(1-T^{*} T / \beta^{2}\right) x, y\right)^{\prime}$ on $H$. The identity function from $\left\{H,(\cdot, \cdot)^{\prime}\right\}$ to $\{H,(\cdot, \cdot)\}$ is continuous. If $\{H,(\cdot, \cdot)\}$ is complete, by Note 5 of [4], the set $Z$ of all $\|\cdot\|^{\prime}$-limits in $H^{\prime}$ of sequences in $H$ with $\|\cdot\|$-limit 0 is closed in $H^{\prime}$ and also an algebraic complement of $H$ in $H^{\prime}$, and if $P$ is the orthogonal projection of $H^{\prime}$ onto $Z^{\perp}$ then $(\cdot, \cdot)$ is equivalent on $H$ to $(P \cdot, P \cdot)^{\prime}$. That is, the inner product $\left(\left(1-T^{*} T / \beta^{2}\right) x, y\right)^{\prime}$ on $H$ is equivalent to the inner product $(P x, P y)^{\prime}$ on $H$, the inner product in $H^{\prime}$ of the shadow of $x$ in $Z^{\perp}$ with the shadow in $Z^{\perp}$ of $y$. Another point of view, starting with a complete space $\left\{H^{\prime},(\cdot, \cdot)^{\prime}\right\}$, an operator $T$ on $\left\{H^{\prime},(\cdot, \cdot)^{\prime}\right\}$, and a dense, proper subspace $H_{1}$ of $H^{\prime}$, and yielding a shadow inner product $\left(\left(1-T^{*} T\right) \cdot, \cdot\right)^{\prime}$ for $H_{1}$ such that $\left\{H_{1},\left(\left(1-T^{*} T\right) \cdot, \cdot\right)^{\prime}\right\}$ is complete, will be pursued in Example 3.

THEOREM 3. Suppose, as in Theorem 2, that $\left\{H^{\prime},(\cdot, \cdot)^{\prime}\right\}$ is a complete inner product space, that $H_{1}$ is a dense subspace of $H^{\prime}$, and that $T$ is an operator on $\left\{H^{\prime},(\cdot, \cdot)^{\prime}\right\}$ such that $T\left(H_{1}\right)$ lies in $H_{1}$. Suppose that $\beta$ is a positive number and that, with $T^{*}$ the adjoint of $T$ in $\left\{H^{\prime},(\cdot, \cdot)^{\prime}\right\}$, (i) $\beta$ is not less than the operator-norm for $T$ in $\left\{H^{\prime},(\cdot, \cdot)^{\prime}\right\}$ and (ii) $1-T^{*} T / \beta^{2}$ is a one-to-one transformation on $H_{1}$. Then for $x$ and $y$ in $H_{1}$ the formula $(x, y)^{\prime \prime}=\left(\left(1-T^{*} T / \beta^{2}\right) x, y\right)^{\prime}$ defines an inner product $(\cdot, \cdot)^{\prime \prime}$ for $H_{1}$ such that if $(\cdot, \cdot)$ denotes $(\cdot, \cdot)^{\prime \prime}$ on $H_{1}$ then for $x$ in $H_{1} \sum_{p=0}^{\infty}\left\|(T / \beta)^{p} x\right\|^{2}$ converges, with limit not exceeding $\left(\|x\|^{\prime}\right)^{2}$. In case $\lim _{p \rightarrow \infty}\left(\left\|(T / \beta)^{p} x\right\|^{\prime}\right)=0$ on $H_{1}$, then on $H_{1}(x, y)^{\prime}=(x, y)_{\beta, T}$ and if, in addition, $\left\{H_{1},(\cdot, \cdot)\right\}$ is complete, so that $\left(1-T^{*} T / \beta^{2}\right)^{1 / 2}\left(H_{1}\right)$ is closed in $H^{\prime}$, and $H^{\prime} \neq H_{1}$ then the restriction of $T$ to $H_{1}$ is not continuous in $\left\{H_{1},(\cdot, \cdot)\right\}$. (Despite the conven- 
tion of the introduction, here $(\cdot, \cdot)$ is not given beforehand).

Proof. Note that, since $1-T^{*} T / \beta^{2}$ is a one-to-one function when restricted to $H_{1},\left\{H_{1},(\cdot, \cdot)^{\prime \prime}\right\}$ is isometrically isomorphic to the subspace $\left(1-T^{*} T / \beta^{2}\right)^{1 / 2}\left(H_{1}\right)$ of $\left\{H^{\prime},(\cdot, \cdot)^{\prime}\right\}$. Thus, writing $(\cdot, \cdot)$ in place of $(\cdot, \cdot)^{\prime \prime},\left\{H_{1},(\cdot, \cdot)\right\}$ is complete if and only if $\left(1-T^{*} T / \beta^{2}\right)^{1 / 2}\left(H_{1}\right)$ is closed in $H^{\prime}$. Suppose $n$ is a positive integer and each of $x$ and $y$ is in $H_{1}$. We have

$$
\begin{aligned}
\sum_{p=0}^{n}\left((T / \beta)^{p} x,(T / \beta)^{p} y\right) \\
=\sum_{p=0}^{n}\left((T / \beta)^{p} x,(T / \beta)^{p} y\right)^{\prime} \\
\quad-\sum_{p=0}^{n}\left((T / \beta)^{p+1} x,(T / \beta)^{p+1} y\right)^{\prime} \\
=(x, y)^{\prime}-\left((T / \beta)^{n+1} x,(T / \beta)^{n+1} y\right)^{\prime} .
\end{aligned}
$$

Hence, in case $\lim _{p \rightarrow \infty}\left\|(T / \beta)^{p} x\right\|^{\prime}=0$ on $H_{1}$ then on $H_{1}(x, y)^{\prime}=$ $(x, y)_{\beta, T}$. Now for $x$ in $H_{1}$ the number-sequence $\left\{\left\|(T / \beta)^{p} x\right\|^{\prime}\right\}_{p=0}^{\infty}$ is nonincreasing with limit $\alpha_{x}$. By (C), for $x$ in $H_{1}$

$$
\begin{aligned}
\sum_{p=0}^{\infty} \| & (T / \beta)^{p} x \|^{2} \\
& =\left(\|x\|^{\prime}\right)^{2}-\left(\alpha_{x}\right)^{2} \leqq\left(\|x\|^{\prime}\right)^{2} .
\end{aligned}
$$

Suppose $H^{\prime} \neq H_{1},(x, y)^{\prime}=(x, y)_{\beta, T}$ on $H_{1}$, and $\left\{H_{1},(\cdot, \cdot)\right\}$ is complete. Then, by Lemma 1 , in case $T$ on $H_{1}$ is continuous in $\left\{H_{1},(\cdot, \cdot)\right\}$, $\left\{H_{1},(\cdot, \cdot)^{\prime}\right\}$ is complete, so that $H_{1}$ is closed in $H^{\prime}$. Since $H_{1}$ is dense in $H^{\prime}$ and $H_{1} \neq H^{\prime}, H_{1}$ is not closed in $H^{\prime}$. Hence, $T$ on $H_{1}$ is not continuous in $\left\{H_{1},(\cdot, \cdot)\right\}$.

EXAMPLE 3. Suppose that on $l^{2}\langle f, g\rangle=\sum_{p=0}^{\infty} f_{p} g_{p}^{*}$ and that $y$ is the point of $l^{2}$ such that $y_{0}=1$ and for $p>0 y_{p}=0$. Suppose $Y$ is the linear span of $\{y\}, P$ the orthogonal projection of $l^{2}$ onto $Y^{\perp}$, and $T$ the operator on $l^{2}$ such that $T(c)$ is the sequence $d$, with $d_{0}=\sum_{p=1}^{\infty} c_{p} / 2^{p+1}, d_{1}=c_{0}$, and for $p>1 d_{p}=c_{p-1} / 2^{2 p-1}$. Now $T^{*}(c)$ is the sequence $e$ such that $e_{0}=c_{1}$ and for $p>0 e_{p}=c_{0} / 2^{p+1}+c_{p+1} / 2^{2 p+1}$ and $T^{*} T(c)$ the sequence $f$ such that $f_{0}=c_{0}$ and for $p>0 f_{p}=$ $\left[\sum_{p=1}^{\infty} c_{q} / 2^{q+1}\right] / 2^{p+1}+c_{p} / 2^{4 p+2}$. Hence,

$$
\begin{aligned}
& \left\langle\left(1-T^{*} T\right) c, c\right\rangle \\
& \quad=\sum_{p=1}^{\infty}\left[1-1 / 2^{4 p+2}\right]\left|c_{p}\right|^{2}-\sum_{p=1}^{\infty}\left\{\left[\sum_{q=1}^{\infty} c_{q} / 2^{q+1}\right] c_{p}^{*} / 2^{p+1}\right\} \\
& \quad=\sum_{p=1}^{\infty}\left[1-1 / 2^{4 p+2}\right]\left|c_{p}\right|^{2}-\left|\sum_{p=1}^{\infty} c_{p} / 2^{p+1}\right|^{2} \\
& \quad \geqq(63 / 64) \sum_{p=1}^{\infty}\left|c_{p}\right|^{2}-\left[\sum_{p=1}^{\infty}\left|c_{p}\right|^{2}\right]\left[\sum_{p=1}^{\infty} 1 / 2^{2 p+2}\right]
\end{aligned}
$$




$$
\geqq(1 / 2) \sum_{p=1}^{\infty}\left|c_{p}\right|^{2} \text {. }
$$

By the above inequality,

$$
\langle P c, P c\rangle \geqq\left\langle\left(1-T^{*} T\right) c, c\right\rangle \geqq(1 / 2)\langle P c, P c\rangle .
$$

Since $\langle c, c\rangle-\langle T c, T c\rangle \geqq 0$ on $l^{2}$, the operator-norm for $T$ does not exceed 1. However, $T^{2}(c)=g$, where $g_{0}=c_{0} / 4+\sum_{p=2}^{\infty}\left(c_{p-1}\right) / 2^{3 p}$, $g_{1}=\sum_{p=1}^{\infty} c_{p} / 2^{p+1}, g_{2}=c_{0} / 8$, and for $p>2 g_{p}=\left(c_{p-2}\right) / 2^{4 p-4}$. Computation reveals that the operator-norm for $T^{2}$ does not exceed 1/2. Hence, $\lim _{p \rightarrow \infty}\left\langle T^{p} c, T^{p} c\right\rangle$ is 0 on $l^{2}$. Note that $T\left(l^{2}\right) \cap Y$ is $\{0\}$. Also, with $z$ the $l^{2}$-sequence such that for $p \geqq 0 \quad z_{p}$ is the sequence $w$ with $w_{q}=2^{p+1}$ or 0 accordingly as $q=p$ or not, $T z$ has limit $y$ in $l^{2}$. Hence, $y$ is in $\overline{T\left(l^{2}\right)}$. Since $\overline{P T\left(l^{2}\right)}$ is $Y^{\perp}$, we conclude that $T\left(l^{2}\right)$ is dense in $l^{2}$.

Suppose $H_{1}$ is an algebraic complement of $Y$ in $l^{2}$ and $T\left(l^{2}\right)$ is a subspace of $H_{1}$. Then the formula $(x, y)^{\prime \prime}=\langle P x, P y\rangle$ defines an inner product for $H_{1}$ such that $\left\{H_{1},(\cdot, \cdot)^{\prime \prime}\right\}$ is complete. By (D), the formula $(x, y)=\left\langle\left(1-T^{*} T\right) x, y\right\rangle$ defines an inner product for $H_{1}$ equivalent to $(\cdot, \cdot)^{\prime \prime}$. Of course, with $\beta=1$, by Theorem $3\langle\cdot, \cdot\rangle=$ $(\cdot, \cdot)_{\beta, T}$ on $H_{1}$. It is of interest to note that $\left[(x, y)^{\prime \prime}\right]_{\beta, T}$ $\left(=\sum_{p=0}^{\infty}\left\langle P T^{p} x, P T^{p} y\right\rangle\right)$ is equivalent to $\langle\cdot, \cdot\rangle$ on $H_{1}$. For

$$
(1 / 2)\left[\|x\|^{\prime \prime}\right]^{2} \leqq\|x\|^{2} \leqq\left[\left\|x^{\prime \prime}\right\|^{2}\right]
$$

implies

$$
(1 / 2)\left[(x, x)^{\prime \prime}\right]_{\beta, T} \leqq(x, x)_{\beta, T} \leqq\left[(x, x)^{\prime \prime}\right]_{\beta, T}
$$

on $H_{1}$.

Note 1. An argument for most of the following, known to the author through work of MacNerney [6], may be found in [1] (Lemma, p. 316), in which it is partly attributed to Friedrichs [3]. No argument will be offered here.

Suppose $\left\{H_{1},(\cdot, \cdot)^{\prime}\right\}$ is complete and continuously situated in $\{H,(\cdot, \cdot)\}$, in the sense that $H_{1}$ lies in $H$ and there is a positive number $c$ such that $\|\cdot\| \leqq c\|\cdot\|^{\prime}$ on $H_{1}$, that $H_{1}$ is dense in $H$, and that $B$ is the adjoint of the identity function from $\left\{H_{1},(\cdot, \cdot)^{\prime}\right\}$ to $\{H,(\cdot, \cdot)\}$, so that $B$ is that linear transformation from $H$ to $H_{1}$ such that for $x$ in $H_{1}$ and $y$ in $H(x, y)=(x, B y)^{\prime}$. Suppose $C$ is an operator on $\{H,(\cdot, \cdot)\}$. Then

(1) $B$ is positive definite in $\{H,(\cdot, \cdot)\}$ and the operator-norm for $B$ in $\{H,(\cdot, \cdot)\}$ does not exceed $c$;

(2) with $B^{1 / 2}$ the positive definite square-root of $B$ in $\{H,(\cdot, \cdot)\}$ 
and $B^{-1 / 2}=\left(B^{1 / 2}\right)^{-1}, H_{1}=B^{1 / 2}(H)$ and $(\cdot, \cdot)^{\prime}=\left(B^{-1 / 2} \cdot, B^{-1 / 2} \cdot\right)$ on $H_{1}$;

(3) if $C(H)$ lies in $H_{1}$ then $C$ is continuous from $\{H,(\cdot, \cdot)\}$ to $\left\{H_{1},(\cdot, \cdot)^{\prime}\right\}$;

(4) if $C B=B C$, then $C B^{1 / 2}=B^{1 / 2} C$ so that $C\left(H_{1}\right)$ lies in $H_{1}$ and for $x$ and $y$ in $H$, with $x \neq 0,\left\|C B^{1 / 2} x\right\|^{\prime} /\left\|B^{1 / 2} x\right\|^{\prime}=\|C x\| /\|x\|$ and $\left(C B^{1 / 2} x, B^{1 / 2} y\right)^{\prime}=(C x, y)$; hence, the operator-norm in $\left\{H_{1},(\cdot, \cdot)^{\prime}\right\}$ for the restriction $C_{1}$ of $C$ to $H_{1}$ is the operator-norm for $C$ in $\{H,(\cdot, \cdot)\}$ and if $C$ is nonnegative in $\{H,(\cdot, \cdot)\} C_{1}$ is nonnegative in $\left\{H_{1},(\cdot, \cdot)^{\prime}\right\}$; and (5) if $C(H)$ is dense in $H$ and $C$ is one-to-one the formula $(x, y)^{\prime \prime}=\left(C^{-1} x, C^{-1} y\right)$ defines an inner product for $C(H)$ such that $\left\{C(H),(\cdot, \cdot)^{\prime \prime}\right\}$ is complete and continuously situated in $\{H,(\cdot, \cdot)\}$ and the adjoint of the identity function from $\left\{C(H),(\cdot, \cdot)^{\prime \prime}\right\}$ to $\{H,(\cdot, \cdot)\}$ is $C C^{*}$ on $H$, where $C^{*}$ is the adjoint of $C$ as an operator of $H$ into itself. Moreover, for the adjoint $C^{+}: C(H) \rightarrow H$ of $C: H \rightarrow C(H)$ we have $C C^{*}=C^{+} C$ (or $C^{+}=C C^{*} C^{-1}$ ).

TheOREM 4. Suppose that $H_{1}$ is a dense subspace of $H$. Then in order that $(\cdot, \cdot)_{1}$ be such an inner product for $H_{1}$ that $\left\{H_{1},(\cdot, \cdot)_{1}\right\}$ is complete and continuously situated in $\{H,(\cdot, \cdot)\}$ it is necessary and sufficient that for some operator $C$ on $\{H,(\cdot, \cdot)\}$ and positive number $d H_{1}$ is the set of all $x$ in $H$ such that $\sum_{p=0}^{\infty}\left\|C^{p} x\right\|^{2}$ converges and, if each of $x$ and $y$ is in $H_{1},(x, y)_{1}=d \sum_{p=0}^{\infty}\left(C^{p} x, C^{p} y\right)$.

Proof. The sufficiency of the condition follows from Lemma 1. To argue necessity, let $e$ be a number such that for $x$ in $H_{1}\|x\|^{2} \leqq$ $e\left(\|x\|_{1}\right)^{2}$ and $(\cdot, \cdot)^{\prime}$ be $e(\cdot, \cdot)_{1}$ on $H_{1}$. Then the complete inner product space $\left\{H_{1},(\cdot, \cdot)^{\prime}\right\}$ is continuously situated in $\{H,(\cdot, \cdot)\}$ and the operator-norm for the identity function from $\left\{H_{1},(\cdot, \cdot)^{\prime}\right\}$ to $\{H,(\cdot, \cdot)\}$ does not exceed 1 . Hence, with $B$ as in Note 1, the operator-norm for $B$ in $\{H,(\cdot, \cdot)\}$ does not exceed 1 . Suppose that $C$ is $(1-B)^{1 / 2}$ on $H$, so that $B=1-C^{2}$. Since $B C=C B$, by Note $1 C\left(H_{1}\right)$ lies in $H_{1}$, the restriction of $C$ to $H_{1}$ is nonnegative in $\left\{H_{1},(\cdot, \cdot)^{\prime}\right\}$, and the operator-norm for this restriction in $\left\{H_{1},(\cdot, \cdot)^{\prime}\right\}$, does not exceed 1. By Theorem 3, $\sum_{p=0}^{\infty}\left\|C^{p} x\right\|^{2}$ converges on $H_{1}$. (Note that $\left\{H^{\prime},(\cdot, \cdot)^{\prime}\right\}$ in Theorem 3 is replaced by $\left\{H_{1},(\cdot, \cdot)^{\prime}\right\}$ here and that $T=C, 1-$ $T^{*} T=B,\left(\left(1-C^{2}\right) x, y\right)^{\prime}=(B x, y)^{\prime}=(x, y)$.) Suppose that $\left\{H^{\prime \prime},(\cdot, \cdot)^{\prime \prime}\right\}$ is the complete inner product space of all $x$ in $H$ for which $\sum_{p=0}^{\infty}\left\|C^{p} x\right\|^{2}$ converges with $(x, y)^{\prime \prime}=\sum_{p=0}^{\infty}\left(C^{p} x, C^{p} y\right)$. Note that, since $H_{1}$ lies in $H^{\prime \prime}, H^{\prime \prime}$ is dense in $H$ and $\left(1-C^{2}\right)(H)$ lies in $H^{\prime \prime}$. Also, by Lemma $1, C\left(H^{\prime \prime}\right)$ lies in $H^{\prime \prime}$ and the restriction of $C$ to $H^{\prime \prime}$ is self-adjoint in $H^{\prime \prime}$. By Note $1,1-C^{2}$ is continuous from $\{H,(\cdot, \cdot)\}$ to $\left\{H^{\prime \prime},(\cdot, \cdot)^{\prime \prime}\right\}$. Suppose each of $x$ and $y$ is in $H^{\prime \prime}$. Then, by Theorem $2,(x, y)=\left(x,\left(1-C^{2}\right) y\right)^{\prime \prime}$. (The $\left\{H^{\prime},(\cdot, \cdot)^{\prime}\right\}$ of Theorem 2 is $\left\{H^{\prime \prime},(\cdot, \cdot)^{\prime \prime}\right\}$ now, $\beta=1$ and $T=C$; the $H_{1}$ of Theorem 2 is $H^{\prime \prime}$ now.) 
Suppose $z$ is in $H, x$ is in $H^{\prime \prime}$, and $y$ is a sequence in $H^{\prime \prime}$ with limit $z$ in $H$. Then

$$
(x, z)=\lim (x, y)=\lim \left(x,\left(1-C^{2}\right) y\right)^{\prime \prime}=\left(x,\left(1-C^{2}\right) z\right)^{\prime \prime},
$$

so that $1-C^{2}$ is the adjoint of the identity function from $\left\{H^{\prime \prime},(\cdot, \cdot)^{\prime \prime}\right\}$ to $\{H,(\cdot, \cdot)\}$. Hence, $H^{\prime \prime}=\left(1-C^{2}\right)^{1 / 2}(H)=H_{1}$ and for $x$ and $y$ in $H_{1}$, by Note 1 ,

$$
\begin{aligned}
(x, y)_{1} & =(1 / e)(x, y)^{\prime} \\
& =(1 / e)\left(\left(1-C^{2}\right)^{-1 / 2} x,\left(1-C^{2}\right)^{-1 / 2} y\right) \\
& =(1 / e)(x, y)^{\prime \prime} \\
& =(1 / e) \sum_{p=0}^{\infty}\left(C^{p} x, C^{p} y\right) .
\end{aligned}
$$

The theorem is established, taking $d$ as $1 / e$.

It may be noted that an argument for Theorem 4 could be based on a theorem, Theorem 2 of [5], of the author and Note 1. The argument given above is more closely related to the other theorems of this paper.

Theorem 5. Suppose that $H_{1}$ is a dense subspace of $H$ and $T$ is a linear transformation from $H_{1}$ to $H_{1}$. Then in order that there be an inner product $(\cdot, \cdot)_{1}$ for $H_{1}$ such that $\left\{H_{1},(\cdot, \cdot)_{1}\right\}$ is complete and continuously situated in $\{H,(\cdot, \cdot)\}$ and $T$ is continuous in $\left\{H_{1},(\cdot, \cdot)_{1}\right\}$ it is necessary and sufficient that for some pair, $\beta$ and $\gamma$, of positive numbers and some operator $C$ on $\{H,(\cdot, \cdot)\} H_{1}$ is the set of all $x$ in $H$ for which $\sum_{p=0}^{\infty}\left\|C^{p} x\right\|^{2}$ converges and for $x$ in $H_{1} \sum_{p=0}^{\infty}\left\|(T / \beta)^{p} x\right\|^{2} \leqq \gamma \sum_{p=0}^{\infty}\left\|C^{p} x\right\|^{2}$.

Proof. To argue necessity, suppose $b$ is the operator-norm for $T$ in $\left\{H_{1},(\cdot, \cdot)_{1}\right\}$ and $\beta=2 b$. By Theorem 4 , there is an operator $C$ in $\{H,(\cdot, \cdot)\}$ and a positive number $d$ such that $H_{1}$ is the set of all $x$ in $H$ for which $\sum_{p=0}^{\infty}\left\|C^{p} x\right\|^{2}$ converges, with limit $(1 / d)\left(\|x\|_{1}\right)^{2}$. Now, with $e=(1 / d)^{1 / 2},\|x\| \leqq e\|x\|_{1}$ and

$$
\begin{aligned}
\sum_{p=0}^{\infty} \| & (T / \beta)^{p} x \|^{2} \leqq e^{2} \sum_{p=0}^{\infty}\left(\left\|(T / \beta)^{p} x\right\|_{1}\right)^{2} \\
& \leqq e^{2}(4 / 3)\left(\|x\|_{1}\right)^{2}=(4 / 3) \sum_{p=0}^{\infty}\left\|C^{p} x\right\|^{2},
\end{aligned}
$$

on $H_{1}$, so that the condition follows with $\gamma=4 / 3$.

To argue the sufficiency of the condition, suppose $(x, y)_{1}=$ $\sum_{p=0}^{\infty}\left(C^{p} x, C^{p} y\right)$ on $H_{1}$, so that $\left\{H_{1},(\cdot, \cdot)_{1}\right\}$ is complete and continuously situated in $\{H,(\cdot, \cdot)\}$, and set $(x, y)_{2}=\sum_{p=0}^{\infty}\left((T / \beta)^{p} x,(T / \beta)^{p} y\right)$ on 
$H_{1}$. Now $T$ on $H_{1}$ is continuous in $\left\{H_{1},(\cdot, \cdot)_{1}\right\}$ and $\|x\|_{2} \leqq \gamma^{1 / 2}\|x\|_{1}$ on $H_{1}$. Suppose $T$ is not continuous in $\left\{H_{1},(\cdot, \cdot)_{1}\right\}$. Then, by the Closed Graph theorem, there is an $H_{1}$-sequence $x$ with limit 0 in $\left\{H_{1},(\cdot, \cdot)_{1}\right\}$ such that $T x$ has limit $y \neq 0$ in $\left\{H_{1},(\cdot, \cdot)_{1}\right\}$. Since $\|z\|_{2} \leqq$ $\gamma^{1 / 2}\|z\|_{1}$ on $H_{1}, x$ has limit 0 , and $T x$ limit $y$, in $\left\{H_{1},(\cdot, \cdot)_{2}\right\}$. But $T x$ has limit 0 in $\left\{H_{1},(\cdot, \cdot)_{2}\right\}$. Thus, $y=0$. This is a contradiction.

EXAMPLE. There is a dense subspace $H_{1}$ of $H$ and a linear transformation $T$ on $H_{1}$ such that $T\left(H_{1}\right)$ lies in $H_{1}$, the formula $(x, y)_{1}=\sum_{p=0}^{\infty}\left(T^{p} x, T^{p} y\right)$ defines on $H_{1}$ an inner product such that $\left\{H_{1},(\cdot, \cdot)_{1}\right\}$ is complete, and yet $T$ is not a closed operator in $\{H,(\cdot, \cdot)\}$.

Suppose $C$ is an operator on $H$ such that the set $H_{2}$ of all $x$ in $H$ for which $\sum_{p=0}^{\infty}\left\|C^{p} x\right\|^{2}$ converges is a dense proper subspace of $H$. Suppose $y$ is not in $H_{2}, H_{1}$ is the linear span of $\{y\}$ and $H_{2}$, and $\phi$ is the algebraic projection of $H_{1}$ onto $H_{2}$ with kernel the linear span $Y$ of $\{y\}$. Suppose $T$ is $C \phi+1 / 2(1-\phi)$ on $H_{1}$. Since $C\left(H_{2}\right)$ lies in $H_{2}, T^{p}$ is $C^{p}$ on $H_{2}$. Since the set of all $x$ for which $\sum_{p=0}^{\infty}\left\|T^{p} x\right\|^{2}$ converges is a linear space including both $Y$ and $H_{2}$, this set is $H_{1}$. Define $(x, y)_{1}$ to be $\sum_{p=0}^{\infty}\left(T^{p} x, T^{p} y\right)$ on $H_{1}$. Then $H_{2}$ is a complete subspace of $\left\{H_{1},(\cdot, \cdot)_{1}\right\}$. Since $Y$ is one-dimensional, $\left\{H_{1},(\cdot, \cdot)_{1}\right\}$ is complete. Now, since $y$ is not in $H_{2}, C y \neq(1 / 2) y$ so that $T$ does not lie in $C$. Yet the closure of $T$ in $H \times H$ includes $C$. Hence, the closure of $T$ in $H \times H$ is not a function.

\section{REFERENCES}

1. R. Adams, N. Aronszajn, and M. S. Hanna, Theory of Bessel potentials, III, Ann. Inst. Fourier (Grenoble), 19 (1969), fasc. 2 (1970), 279-338.

2. P. A. Fillmore and J. P. Williams, On operator ranges, Advances in Math., 7 (1971), 254-281.

3. K. O. Friedrichs, Spektraltheorte halbbeschränkter Operatoren und Anwendung auf die Spektralzerlegung von Differentialoperatoren, Math. Ann., 109 (1934), 465-487, 685-713. Errata: Ibid., 110 (1935), 777-779.

4. George O. Golightly, Graph-dense linear transformations, Pacific J. Math., 82, No. 2 (1979), 371-377.

5. - A characterization of the range of a bounded linear transformation in Hilbert space, Proc. Amer. Math. Soc., 79, no. 4 (1980), 591-592.

6. J. S. Mac Nerney, Continuous embeddings of Hilbert spaces, Rend. Circ. Mat. Palermo,

(2) 19 (1970), 109-112.

7. — Dense embeddings of Hilbert spaces, Proc. Amer. Math. Soc., 24, No. 1 (1970), 92-94.

8. J. D. Maitland-Wright, All operators on a Hilbert space are bounded, Bull. Amer. Math. Soc., 79 (1973), 1247-1250. 



\title{
PACIFIC JOURNAL OF MATHEMATICS
}

\section{EDITORS}

\author{
DONALD BABBITT (Managing Editor) \\ University of California \\ Los Angeles, CA 90024 \\ Hugo Rossi \\ University of Utah \\ Salt Lake City, UT 84112 \\ C. C. MOORE and ARthur Agus \\ University of California \\ Berkeley, CA 94720
}

J. DUGUNDJI

Department of Mathematics

University of Southern California

Los Angeles, CA 90007

R. FINN and J. MILGRAM

Stanford University

Stanford, CA 94305

\section{ASSOCIATE EDITORS}
R. ARENS
E. F. BeCKENBACH
B. H. NeumanN
F. WoLF
K. YoSHIDA

\section{SUPPORTING INSTITUTIONS}

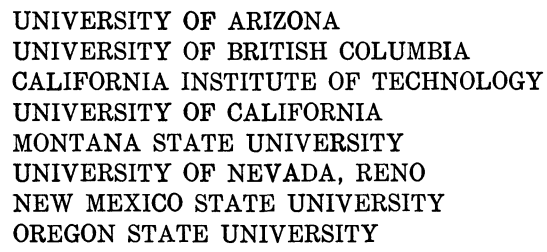

UNIVERSITY OF ARIZONA

UNIVERSITY OF BRITISH COLUMBIA

CALIFORNIA INSTITUTE OF TECHNOLOGY

UNIVERSITY OF CALIFORNIA

MONTANA STATE UNIVERSITY

UNIVERSITY OF NEVADA, RENO

NEW MEXICO STATE UNIVERSITY

OREGON STATE UNIVERSITY

\author{
UNIVERSITY OF OREGON \\ UNIVERSITY OF SOUTHERN CALIFORNIA \\ STANFORD UNIVERSITY \\ UNIVERSITY OF AAWAII \\ UNIVERSITY OF TOKYO \\ UNIVERSITY OF UTAH \\ WASHINGTON STATE UNIVERSITY \\ UNIVERSITY OF WASHINGTON
}

The Supporting Institutions listed above contribute to the cost of publication of this Journal, but they are not owners or publishers and have no responsibility for its content or policies,

Mathematical parers intended for publication in the Pacific Journal of Mathematics should be in typed form or offset-reproduced, (not dittoed), double spaced with large margins. Please do not use built up fractions in the text of the manuscript. However, you may use them in the displayed equations. Underline Greek letters in red, German in green, and script in blue. The first paragraph or two must be capable of being used separately as a synopsis of the entire paper. Please propose a heading for the odd unmbered pages of less than 35 characters. Manuscripts, in triplicate, may be sent to any one of the editors. Please classify according to the scheme of Math. Reviews, Index to Vol. 39. Supply name and address of author to whom proofs should be sent. All other communications should be addressed to the managing editor, or Elaine Barth, University of California, Los Angeles, California, 90024.

50 reprints to each author are provided free for each article, only if page charges have been substantially paid. Additional copies may be obtained at cost in multiples of 50 .

The Pacific Journal of Mathematics is issued monthly as of January 1966, Regular subscription rate: $\$ 114.00$ a year (6 Vol., 12 issues). Special rate: $\$ 57.00$ a year to individual members of supporting institution.

Subscriptions, orders for numbers issued in the last three calendar years, and changes of address shoud be sent to Pacific Journal of Mathematics, P.O. Box 969, Carmel Valley, CA 93924, U.S.A. Old back numbers obtainable from Kraus Periodicals Co., Route 100, Millwood, NY 10546.

PUBLISHED BY PACIFIC JOURNAL OF MATHEMATICS, A NON-PROFIT CORPORATION

Printed at Kokusai Bunken Insatsusha (International Academic Printing Co., Ltd.). 8-8, 3-chome, Takadanobaba, Shinjuku-ku, Tokyo 160, Japan. 


\section{Pacific Journal of Mathematics}

\section{Vol. 103, No. $2 \quad$ April, 1982}

Alberto Alesina and Leonede De Michele, A dichotomy for a class of positive

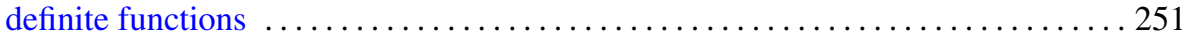

Kahtan Alzubaidy, Rank 2 -groups, $p>3$, and Chern classes . . . . . . . . . . 259

James Arney and Edward A. Bender, Random mappings with constraints on

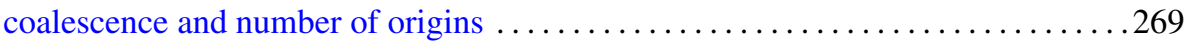

Bruce C. Berndt, An arithmetic Poisson formula . . . . . . . . . . . . . . . 295

Julius Rubin Blum and J. I. Reich, Pointwise ergodic theorems in 1.c.a. groups . . . 301

Jonathan Borwein, A note on $\varepsilon$-subgradients and maximal monotonicity . . . . . . . 307

Andrew Michael Brunner, Edward James Mayland, Jr. and Jonathan Simon,

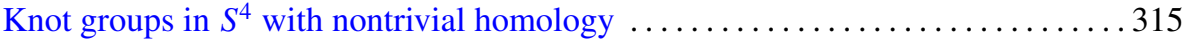

Luis A. Caffarelli, Avner Friedman and Alessandro Torelli, The two-obstacle

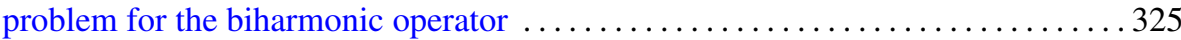

Aleksander Całka, On local isometries of finitely compact metric spaces . . . . . . 337

William S. Cohn, Carleson measures for functions orthogonal to invariant

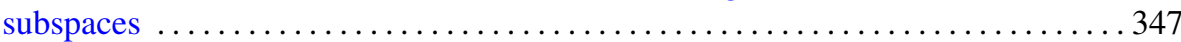

Roger Fenn and Denis Karmen Sjerve, Duality and cohomology for one-relator

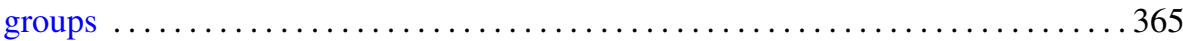

Gen Hua Shi, On the least number of fixed points for infinite complexes . . . . . . . 377

George Golightly, Shadow and inverse-shadow inner products for a class of linear

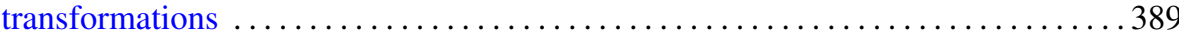

Joachim Georg Hartung, An extension of Sion's minimax theorem with an

application to a method for constrained games $\ldots \ldots \ldots \ldots \ldots \ldots \ldots \ldots \ldots \ldots . \ldots 4$

Vikram Jha and Michael Joseph Kallaher, On the Lorimer-Rahilly and

Johnson-Walker translation planes

Kenneth Richard Johnson, Unitary analogs of generalized Ramanujan sums .

Peter Dexter Johnson, Jr. and R. N. Mohapatra, Best possible results in a class of inequalities

Dieter Jungnickel and Sharad S. Sane, On extensions of nets

Johan Henricus Bernardus Kemperman and Morris Skibinsky, On the

characterization of an interesting property of the arcsin distribution ...

Karl Andrew Kosler, On hereditary rings and Noetherian $V$-rings

William A. Lampe, Congruence lattices of algebras of fixed similarity type. II . . . . 475

M. N. Mishra, N. N. Nayak and Swadeenananda Pattanayak, Strong result for real zeros of random polynomials

Sidney Allen Morris and Peter Robert Nickolas, Locally invariant topologies on free groups

Richard Cole Penney, A Fourier transform theorem on nilmanifolds and nil-theta functions

Andrei Shkalikov, Estimates of meromorphic functions and summability theorems

László Székelyhidi, Note on exponential polynomials

William Thomas Watkins, Homeomorphic classification of certain inverse limit spaces with open bonding maps $\ldots \ldots \ldots \ldots \ldots \ldots \ldots \ldots$

David G. Wright, Countable decompositions of $E^{n}$

Takayuki Kawada, Correction to: "Sample functions of Pólya processes" .

Z. A. Chanturia, Errata: "On the absolute convergence of Fourier series of the 